\title{
Comparison of Mechanical Properties between Carbon/PEEK Composites and Ti Stem for Optimal Design
}

\author{
Sung-Won Yoon ${ }^{1}$, Yun-Hae Kim ${ }^{2 *}$, Min-Kyo Jung ${ }^{2}$, and Ri-ichi Murakami ${ }^{1}$ \\ ${ }^{1}$ Department of Electrical and Computer Engineering, University of Miami, Coral Gables, FL 33124, USA \\ ${ }^{2}$ Department of Industrial and Manufacturing Engineering, Southern Illinois University, Edwardsville, IL 62026-1805, USA \\ ${ }^{3}$ Department of Ocean System Engineering, Jeju National University, Jeju 690-756, Korea
}

(Manuscript Received June 30 2013; Revised July 27, 2013; Accepted August 22, 2013)

\begin{abstract}
This study, a new concept design of the stem and aims to determine the suitability of various carbon/PEEK composite designs for artificial hip joints. Shear stress tested with alternative materials of the Ti-based stem for artificial hip joints. In addition, FEA is conducted according to the fiber ply orientation and the load condition for carbon/PEEK composites. The stem shape of two types was designed through the shape normal of the femur. Multidirectional load cases were used for each FEA model. In the case of general shape, the results show that the stress of ply orientation case II was lower than for cases I and III. On the other hand, in the case of the curved shape, ply orientation case I was lowest. In the case of the Ti stem, the stress of the curved shape was $18 \%$ lower than the general shape.
\end{abstract}

Keywords: Carbon/PEEK Composites, Design, FEA, Stem, Titanium

\section{Introduction}

Recently, research on new medical devices has actively progressed due to the population problem. However, the lifetime of the artificial hip joint being used currently is relatively short.

Over time, the miss ratio of the artificial hip joint considerably increases due to the change in the stress distribution of the bone by stress shielding, dissociation, osteolysis, etc. Therefore, the development of composites for the artificial hip joint is essential through performance evaluation under conditions that it are similar to the human body environment. [1 3]

The generalized metal material is mainstream. Most stems are manufactured from Ti, Co-chrome, or stainless steel alloys. However, metal materials

\footnotetext{
${ }^{*}$ Corresponding author. Tel.: +82-51-410-4355, Fax.: +82-51-410-4350,

E-mail address: yunheak@hhu.ac.kr

Copyright $\odot$ KSOE 2013.
}

deliver small-scale stress to the arbitrary area of the bone due to the difference in rigidity. Thus, composites are the best substitute material to solve this problem. Despite the excellent characteristics of composites, their application to medical devices remains a challenge. [4 6]

Composites in which various designs become available can solve the problems of the existing metal materials. However, it is not easy to implement a design shape because of the different mechanical properties according to the fiber ply orientation. In addition, the complicated shape of the stem has an effect on the distribution of stresses.

In addition, resubstitution is required due to the increased human lifespan. However, the success rate has drastically decreased. Therefore, the development of a patient-customized artificial hip joint is urgently required in order to improve its useful life. $[7,8]$ 
In this study, carbon/PEEK composites are designed to prevent stress concentration from the difference in rigidity and to improve mechanical properties. Two types of patient-customized artificial hip joint stems are designed using a 3D design program. In addition, the stress distribution of the artificial hip joint according to the ply orientation of the fiber is investgated through finite element analysis.

\section{Design and Modeling Method}

\subsection{Method of 3D modeling}

The stem must be designed through the extract of the patient's CT image in order to accurately implement the shape of the customized artificial hip joint stem. However, the size of the femur and the activity environments differ for each patient. Therefore, in this research, a 3D model of the stem was designed from the normal shape, not from a CT image, in order to standardize the femur shape for all patients. The normal shape of the femur is shown in Fig. 1.
In addition, three types of fiber-layered structures were established in order to confirm the distribution of stress and to implement the optimum design of the stem according to the fiber laminating direction.

The mechanical properties of composites and $\mathrm{Ti}$ are shown in Table 1 . In case I, all six plies were oriented at $\left(0^{\circ}\right)\left[\left(0^{\circ}\right)_{6}\right]$. In case II, one ply was oriented at $\left( \pm 45^{\circ}\right)$, one at $\left(0^{\circ} / 90^{\circ}\right)$, and the others at $\left( \pm 45^{\circ}\right)\left[\left( \pm 45^{\circ}\right)_{1}\left(0^{\circ} / 90^{\circ}\right)_{1}\left( \pm 45^{\circ}\right)_{1}\right]$. In case III, all three plies were oriented at $\left( \pm 45^{\circ}\right)\left[\left( \pm 45^{\circ}\right)_{3}\right]$. The carbon/PEEK composites were manufactured by operating the hot press at $380^{\circ} \mathrm{C}$ using the hot press molding method with the prepreg.

\subsection{Design Concept}

The 3D modeling of both stem types of artificial hip joints was designed based on the normal shape of the femur. The first shape follows the shape of the stem made of the existing metal material. The second stem shape is designed for the bottom part of the stem with the curve shaped for an even load dispersion. The designed shape and load direction of the stem are shown in Fig. 2.

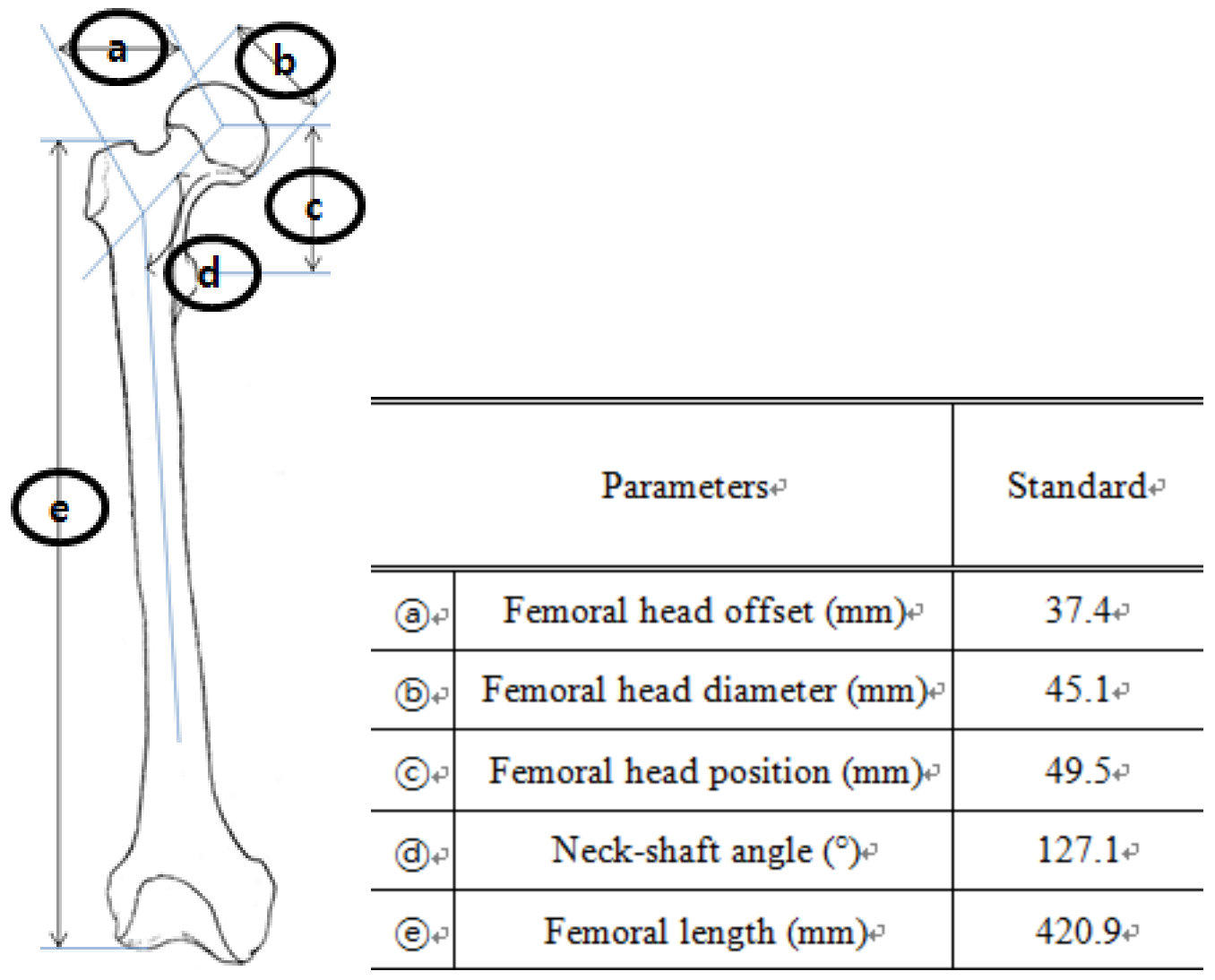

Fig. 1. Standard Shape for Femur 
Table 1. Mechanical Properties of Carbon/PEEK Composites and Ti

\begin{tabular}{|c|c|c|c|c|c|c|c|c|c|}
\hline \multirow{2}{*}{ Constants } & \multicolumn{3}{|c|}{$\begin{array}{l}\text { Modulus of Elasticity } \\
\qquad(\mathrm{GPa})\end{array}$} & \multicolumn{3}{|c|}{$\begin{array}{l}\text { Shear Modulus } \\
\text { (GPa) }\end{array}$} & \multicolumn{3}{|c|}{$\begin{array}{l}\text { Poisson Ratio } \\
\text { (v) }\end{array}$} \\
\hline & $\mathrm{E}_{\mathrm{x}}$ & $\mathrm{E}_{\mathrm{y}}$ & $\mathrm{E}_{\mathrm{z}}$ & $\mathrm{G}_{\mathrm{yz}}$ & $\mathrm{G}_{\mathrm{zx}}$ & $\mathrm{G}_{\mathrm{xy}}$ & $\mathrm{yz}$ & $\mathrm{zX}$ & xy \\
\hline Case I & 95 & 8.5 & 8.5 & 2.5 & 4.5 & 4.5 & 0.3 & 0.3 & 0.3 \\
\hline Case II & 4.5 & 15.5 & 15.5 & 3.5 & 4.0 & 4.0 & 0.3 & 0.3 & 0.3 \\
\hline Case III & 4.0 & 9.8 & 9.8 & 3.0 & 3.5 & 3.5 & 0.3 & 0.3 & 0.3 \\
\hline $\mathrm{Ti}$ & \multicolumn{3}{|c|}{96} & \multicolumn{3}{|c|}{35.3} & \multicolumn{3}{|c|}{0.36} \\
\hline
\end{tabular}

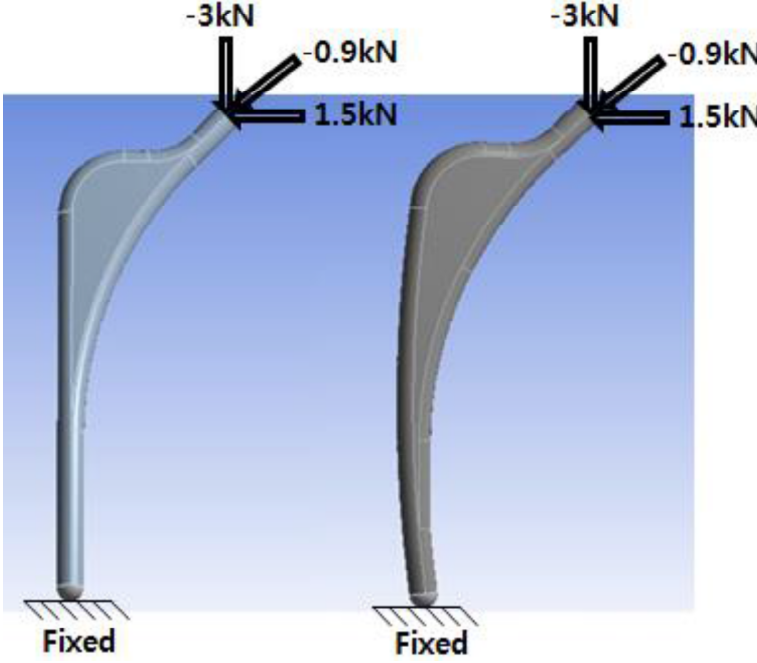

(a) General Shape

(b) Curved Shape

Fig. 2. Design Concept and load direction of the Stem and Ti Stem Composites

\section{Finite Element Analysis}

\subsection{FEA Models}

The meshed shape of the stem is shown in Fig. 3. Details of the model meshes are shown in Table 2. The finite element analysis models were meshed and analyzed using a commercially available program. It is very important to analyze the bio- mechanical characteristics of the geometric design variables before designing the customized artificial hip joint. The femur undergoes various stresses depending on the the lifestyle and posture of the patient. However, it is difficult to reproduce these conditions completely in the simulation. Therefore, the loading condition is applied to the area of neck where the break in the stem mainly occurs. The geometric design variables of the stem were confirmed by using the finite element method. The shear stress of the composites and metal are compared for the artificial hip joint.

\subsection{Loads Conditions}

The stem receives various loads from the muscle. The load condition was determined as shown in Fig. 2 for each of the FEA models. The load applied to the stem head with an angle of $20^{\circ}$ was used to verify the FEA results obtained in the present study by comparing the stresses in the stem and in the $\mathrm{Ti}$ stem model with those reported by previous researchers. The loads were distributed over several nodes to avoid stress concentration and the displacement of all nodes at the distal end of the stem was rigidly constrained. 
Table 2. Details of the meshes for models

\begin{tabular}{c|c|c}
\hline \hline Part & Number of nodes & Number of Elements \\
\hline \hline General Shape (Composites) & 9715 & 7836 \\
\hline Curved Shape (Composites) & 10409 & 8456 \\
\hline General Shape (Metal) & 7382 & 3555 \\
\hline Curved Shape (Metal) & 9824 & 4721 \\
\hline
\end{tabular}

\section{Results and Discussion}

The proposed stem design using carbon/PEEK composites and $\mathrm{Ti}$ was described. Finite element models were used to evaluate the potential of the proposed new design concept. The shear stress of Ti is shown in Fig. 4. The same load was applied to the stem and to the composites. The stress of the curved shape was $18 \%$ lower than the general shape. The proposed stem design is superior to the existing shape as to the stress distribution.

The shear stress according to the ply configuration is shown in Fig. 5. The loading was identical for all experiments. In the case of general shape, the results according to ply configuration show that the shear stress of ply orientation case II were lower than ply orientation cases I and III. On the other hand, in the case of the curved shape, ply orientation case I was lower than the ply orientation case II and III. The mechanical property of ply orientation case I was superior to ply orientation cases II and III due to the curve. Because the stem receives stress perpendicularly the stress applied to the stem was low in ply orientation case I. The distribution of stresses applied to the stem therefore differed according to the fiber ply orientation. Therefore, the fiber ply orientation and the shape of the stem need to be determined by measuring the load accurately since the load applied to the stem differs for each patient.

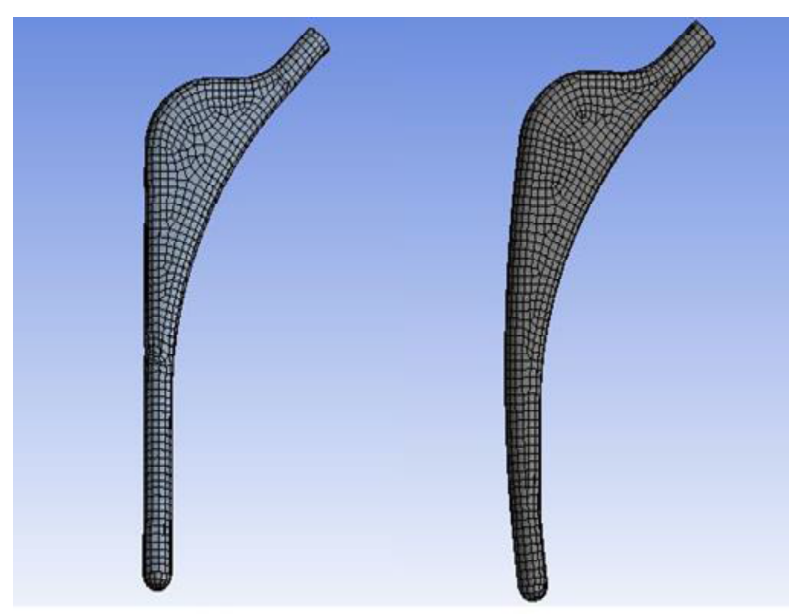

(a) Composites

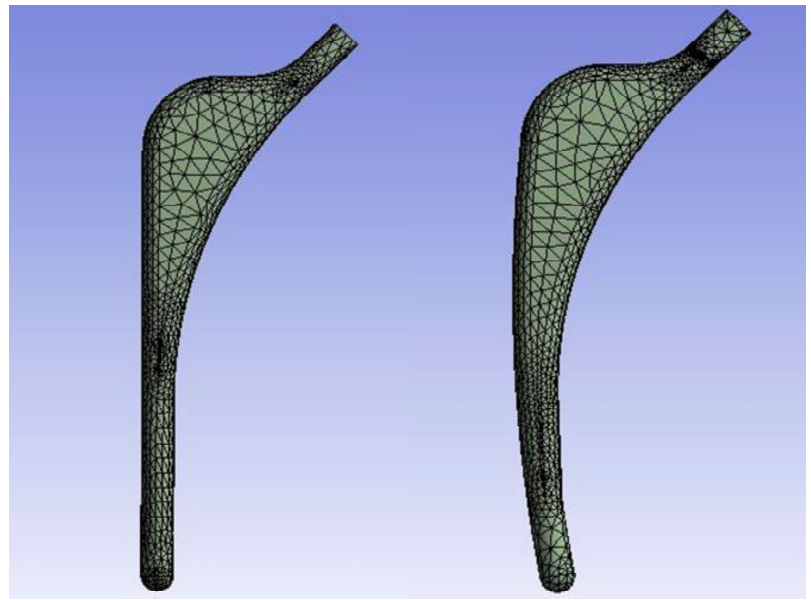

(b) Metal

Fig. 3. Meshed Shape of the Stem 


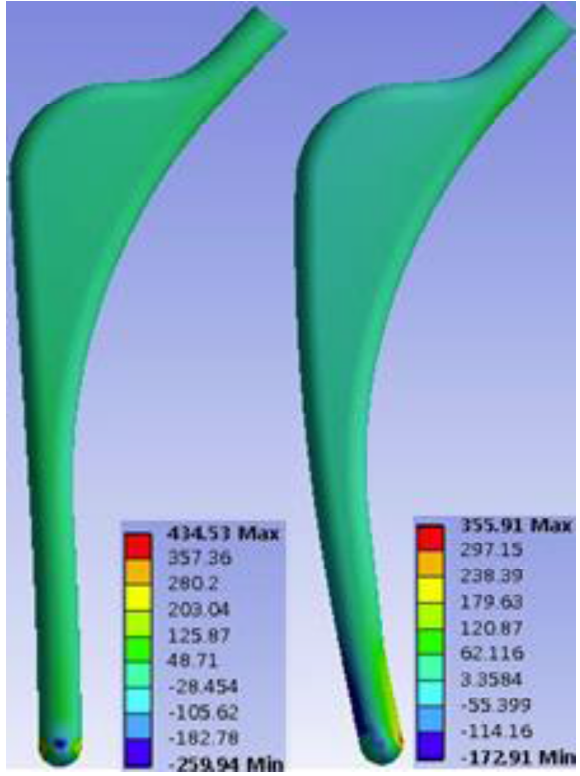

Fig. 4. Shear Stress for Ti

\section{Conclusion}

In this study, two types of stems were designed, and the shear stress of the stems was confirmed through finite element analysis. The stability of the artificial hip joint was determined according to the fiber ply orientation. The following results can be obtained from these data.

(1) The stress of the curved shape was $18 \%$ lower than the general shape. Thus, the proposed stem design is superior to the existing shape as to the distribution of stresses.

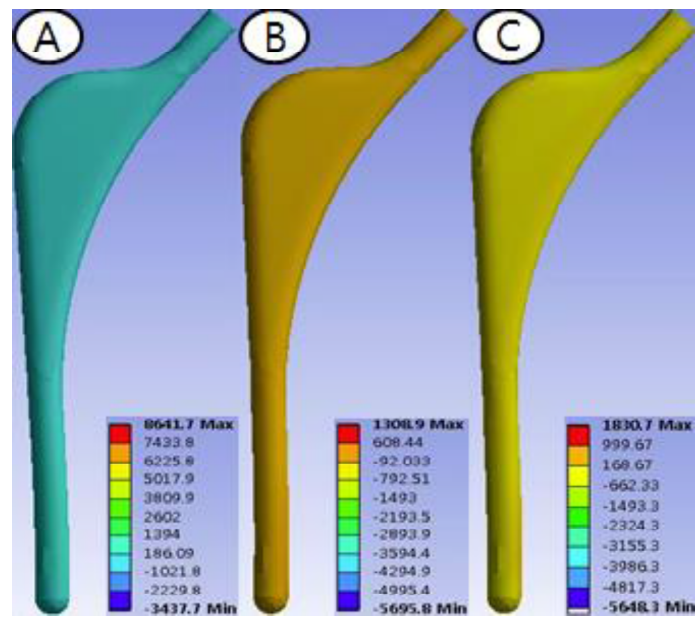

(a) General Shape
(2) In the case of general shape, the shear stress of ply orientation case II was lower than ply orientation cases I and III.

(3) In the case of the curved shape, ply orientation case I was lower than ply orientation cases II and III.

(4) The fiber ply orientation and the shape of the stem need to be determined by measuring the load accurately, because the load applied to the stem differs for each patient.

The customized artificial hip joint is expected to be completed from the analysis performed in this study. It appears that the composites stem can reduce the need for the resubstitution technique.

\section{Acknowledgements}

This work (Grants No. S2046151) was supported by Korean Small Business Innovation Research Program and Research Institute funded Korea Small and Medium Business Administration in 2012.

\section{References}

[1] Y. H. Kim, S. W. Yoon and R. Murakami, Design of Artificial Hip Joint by Carbon/PEEK Composites, Advanced Materials Research, 774-776 (2013) 1336-1341.

[2] Y. H. Kim, S. W. Yoon and R. Murakami, Effect of Moisture Absorption and Fiber Ply Orientation for Artificial Hip Joint on the Mechanical Properties of Carbon/PEEK Composites, Advanced Materials Research, 774-776 (2013) 1326-1335.

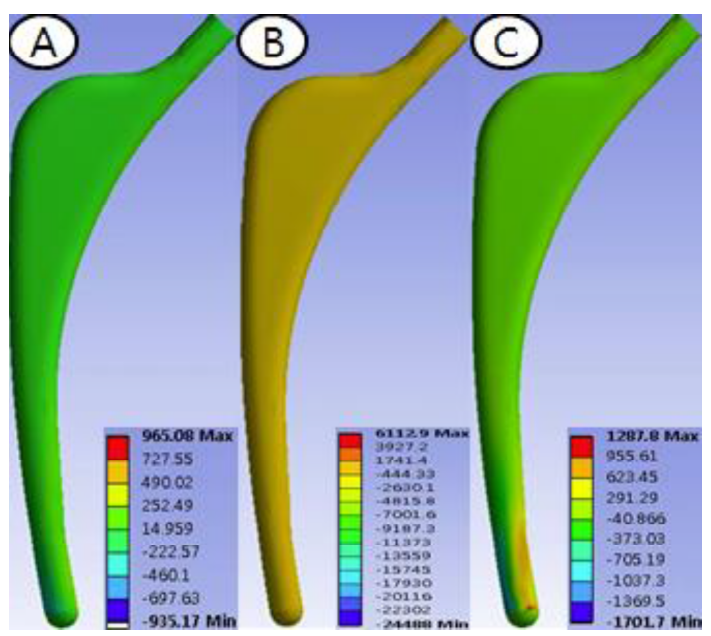

(b) Curved Shape

Fig. 5. Shear Stress by Ply Configuration: (A) ply orientation case I, (B) ply orientation case II, (C) ply orientation case III 
[3] Y. H. Kim, S. W. Yoon and R. Murakami, Mechanical Properties of Carbon/PEEK Composites According to the Fiber Ply Orientation and Sizing Removal of Carbon Fiber for Artificial Hip Joint, Advanced Materials Research, 750-752 (2013) 164-175.

[4] Ikeda M, Komatsu SY, Sowa I, and Niinomi $\mathrm{M}$, Aging behavior of the Ti-29Nb-13Ta-4.6Zr new $b$ alloy for medical implants, Metall Mater Trans A, 33 (2002) 487-493.

[5] Niinomi M, Recent research and development in titanium alloys for biomedical applications and healthcare goods, Sci. Technol. Adv. Mater., 4 (2003) 445-454.
[6] Bania PJ, Lenning GA, and Hall JA, Development and properties of Ti-15V-3Al-3Sn-3Cr. In: Boyer RR, Rosenberg HW, editors. b Titanium Alloys in the 1980's. Warrendale, PA: AIME; (1984) 209-229.

[7] H. Bougherara, M. Bureau, M. Campbell, A. Vadean and L. Yahia, Design of a biomimetic polymer-composite hip prosthesis, Wiley InterScience, (2007) 27-40.

[8] T. Kawamura, M. Zako, T. Kurashiki, H. Nakai, T Furuta, N. Sugano, H. Yoshikawa and S. Bandoh, On a Design Method of Composites Stem Based on CT Images, International Conference on Composite Materials, (2007) $1-10$. 